\title{
Equilibrios macro y general: un asunto por resolver en el mercado de salud en Colombia
}

\author{
Macro and General Equilibrium: \\ an Issue to Resolve on the Market for Health in Colombia
}

Carlos Arturo Meza Carvajalino*

\section{Resumen}

El cambio institucional adoptado por Colombia a partir de la Constitución Política del 91, permitió la creación de nuevos escenarios en la prestación de los servicios públicos. Se creó un mercado de salud, en el cual se han presentado una serie de fallos de mercado e institucionales, originadas por la poca capacidad del SGSSS, para encontrar el equilibrio entre lo macro y lo micro. Lo que ha conducido a que el Gobierno, en su ineficiencia regulatoria, se recargue sobre la institución familia.

\begin{abstract}
La reflexión está soportada sobre las bases de la micro y macrofundamentación económica del equilibrio, y pone en discusión las diferentes variables que afectan el estado de salud, visto como una mercancía, en donde, se rompen los principios de la "ley de Say" y otros elementos de la teoría económica, que no se han tenido en cuenta para que los individuos alcancen su máximo estado de bienestar.
\end{abstract}

Palabras clave: equilibrio general, seguridad social, competencia regulada, fallos de mercado, hogares.

Clasificación JEL: I-11, I-18, I-38.

\footnotetext{
* Economista, Universidad Central, magíster en Ciencias Económicas, Universidad Santo Tomás, magíster en Planificación y Administración del Desarrollo Regional, Universidad de Los Andes. Docente del programa de Maestría en Ciencias Económicas de la Universidad de Santo Tomás. Docente Facultad de Ciencias Económicas y Sociales, Programa de Economía, Universidad de La Salle, director CIHDEP, Universidad de La Salle.
} 


\section{Abstract}

The institutional change adopted by Colombia since the Political Constitution of 91, allowed the creation of new scenarios for the public services. There was created a market of health, in which the government has presented a series of failures of market and institutional faults, originated for small capacity of the SGSSS, to find the balance between macro and microeconomic process. The government has led inefficiency in the processes, su-

\section{Introducción}

Colombia adoptó a partir de la Constitución Política del 91 un sistema de salud mixto bajo la triada Estadomercado-solidaridad, basado en los referentes teóricos universales tanto Bismarkiano (Bismarck, 1883) como Beveridgiano (Beveridge, 1919). El primero propuso un sistema de norma para la seguridad social de tipo contributiva, el cual se fundamentó en la obligatoriedad del pago de cotizaciones por parte de los empleadores y los obreros, regulados por el Estado. El enfoque de seguridad Beveridgiano propone integrar los seguros sociales con la asistencia social y los seguros voluntarios complementarios, los cuales dieron lugar al Seguro Social. Estos elementos, sirvieron de base para la creación del mercado de salud que se puso en marcha a partir de la Ley 100 de 1993.

De igual manera, este mercado está reposa sobre un mercado de derechos ${ }^{1}$, el cual les permite a los agentes contaminadores generar externalidades negativas ${ }^{2}$ (medioam-

1 Artículo 49. La atención de la salud y el saneamiento ambiental son servicios públicos a cargo del Estado.

2 Medioambiente contaminado, por efectos de residuos sólidos que son vertidos a los ríos y chimeneas, entre otros. pported on the bases micro and macroeconomic out of balance, and is tried to put in discussion, the different variables that affect the health, the goods and services, without break the beginning of "Say's law " and other elements of the economic theory, which have not been born in mind the individual welfare.

Key words: general balance, National Health Service, regular competition, failures of market, homes.

Glassification JEL: I-1 1, I-18, I-38.

biente contaminado), cuyo costo es trasladado al estado de salud de las personas, y es ponderado a la sociedad a través de los pagos, que se realizan a través de los aportes parafiscales, tasas e impuestos que servirían en teoría para atenuar el daño causado a las personas (enfermedades) producidas por los agentes externos. Este mercado de derecho se encuentra bajo el lema "quien contamina paga", y es validado en Colombia por la Ley 99 de 1993.

El modelo desde el punto de vista de un mercado de bienes y servicios, se caracteriza por ser un modelo de competencia regulada donde se les delegó al sector privado la responsabilidad social de proveer un servicio público y otros principios que quedaron plasmados en la Constitución Política de Colombia (artículos 48 - 49).

Esta reflexión es un esfuerzo con el que se trata de formalizar un mercado de competencia imperfecta, con los elementos conceptuales del equilibrio general. De igual manera, se pretende entroncar los elementos del merca-

3 El surgimiento del principio "quien contamina paga", también conocido como principio "contaminador-pagador", tiene mucho que ver con lo que el biólogo Garret Hardin llamó: la tragedia de los bienes comunes (Edmunds \& Letey, 1975, p.112). (tomado de Rafael Valenzuela) http://caballeroredverde.blogspot. com/2006/09/el-que-contamina-paga.html 
do de salud en el equilibrio del circuito macroeconómico. Por ello, este artículo se desarrolla en cinco partes, la primera es esta introducción, en la segunda se aborda el tema del circuito macroeconómico y los microfundamentos a partir del enfoque marshalliano. En la tercera, se presenta el mercado de salud y sus fallos de mercado y Estado; en la cuarta parte se presentan algunos elementos que pueden dar lugar a una metodología a partir de los elementos walrasianos de equilibrio general, en situación de competencia imperfecta (fallas de mercado), se presentan algunos elementos que conduzcan a la estimación de un modelo de equilibrio general computable para el sector salud, y que les permita a los hacedores de política encontrar respuestas a la problemática del sistema mixto de competencia regulada, y con ello, encuentren nuevas alternativas distintas a las actuales; por último, en la quinta parte se concluye.

\section{Marco teórico}

A nivel macroeconómico, una situación de equilibrio ${ }^{4}$ se presenta cuando la curva de demanda agregada es igual a la curva de oferta agregada. En términos de una ecuación de identidad (Dornbusch et ál., 2004), se simboliza como $P I B=C+I+G+\bar{X}-\bar{M}$, esto es posible al existir armonía en el flujo que se presenta entre las cantidades agregadas de bienes y servicios, y la estabilidad de los precios en todo el engranaje o circuito económico. En este sentido, el mercado de salud, al entroncarse en el equilibrio macroeconómico, debe soportarse sobre el conjunto de relaciones matemáticas, que se presentan entre las instituciones (hogares, empresas y gobierno), junto a las actividades de producción, consumo, acumulación y resto del mundo; y el flujo de bienes y servicios de salud in-

4 Keynes (1935, p.33) consideró que el volumen ocupación está determinado por la intersección de la función de demanda global y la función de oferta global, porque es este punto donde las expectativas de ganancia del empresario alcanzan su máximo. cluidos en el SGSSS, que hace parte de las imputaciones que se generan tanto en las entradas como en las salidas registradas en la contabilidad nacional.

\subsection{Funcionamiento y equilibrios del circuito económico}

Un circuito económico está conformado, domésticamente, por las cuentas de producción, el consumo y la acumulación. Al agregar a la cuenta del resto del mundo, se generan otros flujos a partir de las variables exportaciones $\bar{X}$ y las importaciones $\bar{M}$, las cuales dan origen a la balanza comercial $(\bar{X}-\bar{M})$.

En donde $Y=Y I N_{f}$ corresponde al pago de la renta de factores; $C$ es el consumo; $T$ equivale a los impuestos indirectos menos los subsidios $I I-S S$; $C C f$ es igual al Consumo de Capital Fijo.

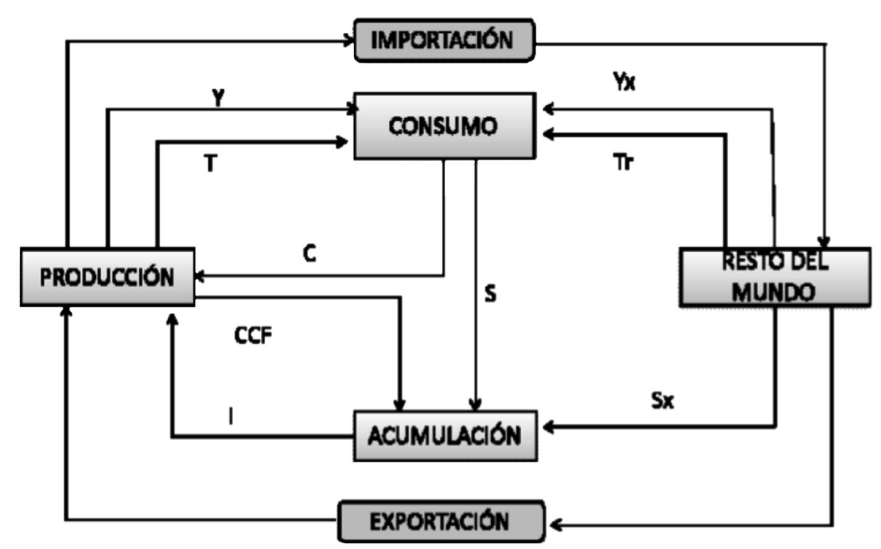

Figura 1. Circuito económico.

Diseño Autor: Fuente Lora: Técnicas de medición económica.

Por otra parte, $S$ y $S_{x}$ son el ahorro doméstico y externo respectivamente; $\mathcal{T}_{r}$ son las transferencias corrientes netas 
del exterior, $I$ es igual a la inversión; los ingresos netos de factores del exterior están representados por $Y_{x}$. Este circuito da lugar al intercambio de mercancías a nivel interno y externo.

Villar (2006) considera que en los circuitos económicos, los miles de agentes que participan de la actividad por su parte son:

Los consumidores demandan bienes y servicios y ofertan distintos tipos de trabajo; las empresas producen mercancías mediante empleo de diversas técnicas de recursos productivos; el Gobierno determina la estructura legal de derechos de propiedad y participa en la economía mediante impuestos y subvenciones, y como demandante y oferente de determinados bienes y servicios.

\subsubsection{Relaciones matemáticas de identidad en el circuito económico}

Con la estimación de identidades se trata de poner en escena la problemática de los elementos fundamentales del modelo de seguridad social en salud. En términos generales, un modelo en economía se define como una aproximación de la realidad. Estas aproximaciones corresponden a un conjunto de relaciones matemáticas, las cuales son abstracciones o simplificaciones que deben expresar las características básicas y esenciales de: i) orden institucional y legal, el cual está soportado en la Constitución Política del 91 y su correspondientes leyes afines como la Ley 100 de 1993, la Ley 99 de 1993 y la 715 de 2001, entre otras; ii) el comportamiento de los agentes en la actividad económica, que son los sujetos que demandan y ofertan servicios y bienes del sistema de salud; iii) las tecnologías incorporadas en los procesos productivos, que corresponden a funciones de producción y de costos de las empresas, representados en los factores de producción (mano de obra, capital, tecnología) y sus correspondientes productividades marginales.

Estas estructuras matemáticas se macrofundamentan en ecuaciones de identidad que van a servir de apoyo logístico y soporte, para estimar las ecuaciones expresadas en i); ii) y iii). En este sentido, a partir del circuito económico se deducen las ecuaciones de identidad y/o equilibrios contables. Se recurre a la contabilidad del sistema de partida doble (entrada y salida). Si se observa en el esquema 1, hay un flujo, el cual se sigue en dirección de las flechas. En este caso hay flechas que entran a las cuentas y flechas que salen de estas.

Tabla 1. Deducción de las ecuaciones de identidad

\begin{tabular}{llll}
\multicolumn{1}{c}{ Guentas } & \multicolumn{1}{c}{ Salidas } & \multicolumn{1}{c}{ Entrada } & \multicolumn{1}{c}{ Ecuación } \\
Producción & {$\left[\right.$ Yin $\left._{f}+(I I-S S)+C C F+M\right]$} & $(I+C+X)$ & Yin $_{f}=C+I+C C F+X-M-(I I-S S)$ \\
Consumo o de instituciones & $S+G$ & $Y_{f}+(I I-S S)+Y_{X}+T_{R}$ & $Y_{f}=S+C-(I I-S S)-Y_{X}-T_{R}$ \\
\hline Acumulación o de la capítal & $I$ & $S+S_{X}+C C F$ & $I=S+S_{X}+C C F$ \\
Resto del mundo & $T_{R}+Y_{X}+S_{X}+X$ & $M$ & $M=T_{R}+Y_{X}+S_{X}+X$
\end{tabular}

Fuente: deducciones del autor. 
Todas estas ecuaciones y actividades, producto de interrelaciones son soporte, para comprender y formalizar modelos que deben engranar con los microfundamentos de un modelo de equilibrio general. A la vez, estos circuitos, se convierten en un proceso complejo, de interrelaciones que permite ver el sistema económico en su conjunto.

Estos sistemas de engranaje de ecuaciones simultáneas, permiten la creación del equilibrio económico general, el cual afecta la cuenta de ingresos y gastos (contribuciones a la seguridad social y prestaciones a la seguridad social) en sus entradas y salidas de acuerdo con el Sistema de Guentas Nacionales del DANE 5 .

\subsection{Modelos de equilibrio general}

La ciencia económica reconoce a Walras (1874), como el pionero en formular un modelo de equilibrio general, quién a través de un sistema de ecuaciones simultáneas, consideró que por lo menos debía existir un equilibrio entre la oferta $Q_{s}$ y la demanda $Q_{d}$ de ahí, que si $Q_{s}$ o $Q_{d}$ se modifican ponen en peligro el punto de equilibrio. En estos términos Lozano et ál (1999), señala que "Walras y sus seguidores percibieron que su teoría sería poco sólida sin un argumento que soportara la existencia de por lo menos un equilibrio".

Ahora, al formalizarse un modelo de ecuaciones simultáneo, de correspondencia con los precios y las cantidades ofrecidas y demandadas, debió suponerse la doble direccionalidad, entre estos precios y las cantidades ofrecidas y demandadas, es decir que $Q=f(p)$ y $P=f(q)$, toda vez que la construcción de estos modelos económicos, se consideren completamente contenidos y si tienen solución única. Esto exige el cumplimiento, de acuerdo con
Dagum et ál. (1983) de dos condiciones, de una necesaria y una necesaria y suficiente.

Las exigencias anteriores suponen que los precios sean una variable endógena, toda vez que la condición necesaria establezca que el número de variables endógenas debe ser igual al número de ecuaciones y el cumplimiento de la condición necesaria y suficiente exige que la matriz de coeficientes, su determinante sea no singular. No obstante, en los comienzos del análisis de equilibrio general señala $\operatorname{Varian}^{6}$ que:

Dado que el número de ecuaciones era igual al número de incógnitas, se concluyó que debía existir una solución en la que se cumplieran todas las ecuaciones. Pero, pronto se descubrió que este argumento era incorrecto. El mero recuento del número de ecuaciones y de incógnitas no es suficiente para demostrar que existe una solución de equilibrio. Y por ello, hay que valerse de instrumentos matemáticos que den lugar a esto.

En estos términos Lozano et ál. (1999), argumenta que:

Para Walras era clara la correspondencia que existía entre el número de precios por determinar y el número de ecuaciones que expresan la igual entre la oferta y la demanda. Pero esto no era suficiente para determinar el equilibrio, ya que las ecuaciones podrían no ser independientes y sólo los precios relativos afectarían el comportamiento de los agentes.

\subsubsection{Elementos walrasianos en desarrollo de un modelo de equilibrio general}

Se entiende de acuerdo con Villar (2006) que los agentes económicos "son las unidades de decisión del modelo". Estos agentes lo que pretenden es maximizar sus función objetivo individual sobre el conjunto de oportunidades. Un caso especial de equilibrio general es el denominado 
intercambio puro $^{7}$. Una economía de intercambio puro se expresa como $E=\left(\boldsymbol{R}_{+}^{\ell}, \gtrless_{i}, \omega_{i}\right)_{i=1}^{m}$ donde, $\boldsymbol{R}_{+}^{\ell}$ corresponde al espacio $^{8}$ de las mercancías; $\gtrsim_{i}$ representa las preferencias del $i$-ésimo consumidor, definidas sobre su plan de consumo; $X \subset \boldsymbol{R}_{+}^{\ell}$; $\omega_{i}$ son los recursos inicialmente disponibles, esto es $\omega_{i} \in \boldsymbol{R}_{+}^{\ell ;} m$ es igual un número finito de consumidores que intercambian unas dotaciones de mercancías dadas exógenamente.

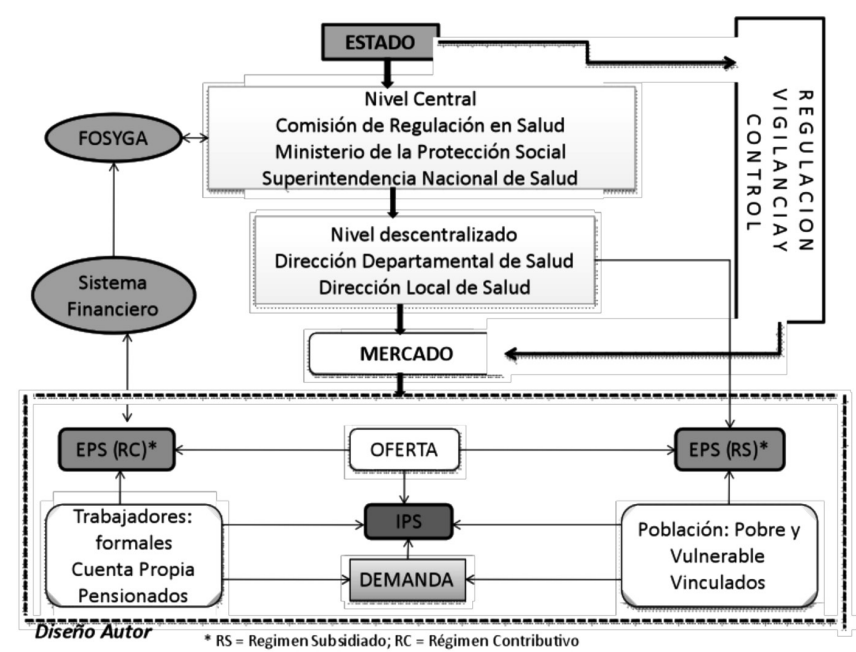

Figura 2. Operación del SGSSS.

Para el análisis, se parte de los supuestos de la existencia de dos consumidores $A$ y $B$, y estos son precio ${ }^{9}$ aceptantes, quienes consumen dos mercancías ${ }^{10}$ (Mas-Colell et ál., 1995) (dos bienes), bajo competencia perfecta. En otros términos los precios serán vectores de $\boldsymbol{R}_{+}^{\ell}$, es de-

7 Economías en las que la única actividad económica es el intercambio y el consumo (Villar, 2006)

8 Espacio Euclídeo dimensional

9 También hay precios negativos y precios neutros. Los negativos, para el caso que compete como se trata de salud de manera integral, el aire contaminado tendrá un precio negativo, los precios neutros son los precios de los bienes públicos gratuitos.

10 Microeconomic Theory. cir, $p \in \boldsymbol{R}_{+}^{\ell}$. Como lo señala Villar (2006), el intercambio puro $E$, se entiende como un mecanismo de asignación de recursos que dependerá del marco institucional en que éste opere (en particular de los derechos de propiedad y del conjunto de reglas que definan que tipo de actuaciones o comportamiento de los agentes son posibles). Obsérvese que estos elementos están implícitos en el SGSSS, esas asignaciones las realizan los agentes privados, regulados por la estacionalidad a través de los marcos regulatorios establecidos a partir de la Constitución del 91.

\subsubsection{Equilibrio walrasiano en un ambiente de competencia imperfecta}

Como ya es sabido, el equilibrio general se plantea bajo un escenario de competencia perfecta. Sin embargo, Lozano et ál. (1999), considera que es claro que ningún mercado satisface plenamente con estos postulados, todo lo contrario ni siquiera se acercan a esta descripción. No obstante, bajo el escenario de competencia imperfecta o fallas de mercado, en un ambiente walrasiano, se "buscan capturar alguna imperfección que parece ser más realista que la hipótesis competitiva, pero dentro de una estructura walrasiana". Esto da lugar a que estas reflexiones se acerquen a la microfundamentación soportada por este enfoque, los cuales se ponen en consideración en el SGSSS.

\section{Circuito y operación del SGSSS}

Como se mencionó en la introducción, el mercado de la salud en Colombia se soporta sobre la triada Estadomercado-solidaridad. Este último pilar es producto de los enfoques universalistas concebidos en Inglaterra por Beveridge y en Alemania por Bismark, el cual se soportó sobre la base de cotizaciones para la obtención de un derecho. Para efectos de análisis se parte de la figura 2, don- 
de se presentan los agentes que intervienen en el mercado privado, en la prestación del servicio público de salud.

Se parte del principio de que el servicio y prestación del servicio de salud es público y se le da el carácter de mercancía, toda vez que se negocia en un mercado, dado una restricción presupuestal. En estos términos, una mercancía es un bien y/o servicio público, "cuando su consumo por parte de una agente no reduce la cantidad disponible para el consumo de los demás". Estos bienes y/o servicios, pueden ser suministrados tanto por empresas privadas como por empresas públicas. Así pues, señala Villar (2006), "que la calificación de bien privado o público tiene que ver con la naturaleza del bien y no con el tipo de empresa o institución que lo suministra".

\subsection{El Estado}

De acuerdo con el SGSSS, el Estado realiza su papel de regulador, el cual se soportan sobre tres organismos: un rector en cabeza del Ministerio de la Protección Social ${ }^{11}$, la Comisión Reguladora en Salud (CRES) ${ }^{12}$, quien crea los acuerdos regulatorios y establece el valor de la UPC y otras funciones de ley y la Superintendencia Nacional de Salud que se encarga de la vigilancia y control.

\subsection{El mercado}

Esta institución, desde el punto de vista de un mercado de bienes y servicios, está conformada por oferentes privados que aprovisionan el servicio público de salud y por los demandantes de estos servicios. El análisis de esta mixtura

11 Ley 1122 de 2007. (enero 9) por la cual se hacen algunas modificaciones en el. Sistema General de Seguridad Social en Salud y se dictan otras disposición.

12 El artículo 3 de la Ley 1122 de 2007, señala que: El CNSSS, mantendrá vigentes sus funciones establecidas en la Ley 100 de 1993, mientras no entre en funcionamiento la CRES.
Estado-mercado, se realizará bajo ejemplos hipotéticos de dos agentes $A$ y $B$, que toman sus planes y decisiones de consumo $x_{i}=\left(x_{i 1}, x_{i 2}, \cdots x_{i \ell}\right)$ en función de su tipo de enfermedad y de servicios que se requieran para maximizar su salud. Ciertamente, como lo manifiesta Stiglitz (2002).

Los servicios médicos, son diferentes al de ropa, de las películas, de los automóviles y de casi todos los demás bienes, y por lo tanto no deben ser controlados por el mercado. La idea de que hay bienes y servicios, como la asistencia sanitaria, que debe suministrarse a todas las personas independientes de su renta, se le conoce con el nombre de igualitarismo específico.

A diferencia de otros bienes como la camisa, en donde los consumidores si no la consumen, máximo les generará una desutilidad momentánea, mientras se le quita el afán propiciado, por ejemplo, por la moda, entonces esta mercancía puede ser sustituta perfecta con otra camisa. No obstante, el estado de salud no puede ser reemplazado por otro estado de salud, entendido como una mercancía que es un bien que genera soluciones de esquina, es decir que el estado de salud frente a otros bienes, el individuo "en algunas situaciones como estas, las preferencias de los individuos pueden ser tales que alcanzan la utilidad máxima al optar por no consumir nada de uno de los bienes". Nicholson (2007). Ejemplo, para maximizar el estado de salud de un enfermo de la vesícula, éste ya no puede escoger entre un bien u otro, puesto que si no se le realiza una cirugía u otro procedimiento que conduzca a eliminar el dolor, es posible que lo lleve a la muerte o la discapacidad.

Por otra parte, Castaño (2000) considera que las enfermedades no se eligen, como los otros bienes, estos pueden considerarse como males y su aparición no es previsible. Estos argumentos son los que llevan a pensar que el bien salud es una mercancía muy especial, la cual se le puede considerar como un bien meritorio, es decir, a cualquier per- 
sona se le debe dar el derecho sin importar su capacidad de pago, concepto que coincide con el igualitarismo especifico.

\subsubsection{La demanda}

Dentro del contexto del mercado de salud en Colombia, el SGSSS cuenta con elementos de una economía de intercambios, en el cual participan $m$ consumidores y $\ell$ mercancías, en donde cada consumidor por su condición de salud, se caracteriza por su conjunto de consumo $x_{i}=\left(x_{i 1}, x_{i 2}, \cdots x_{i \ell}\right) \in \boldsymbol{R}_{+}^{\ell}$, su función de utilidad $\mu_{i}: \boldsymbol{R}_{+}^{\ell} \rightarrow \boldsymbol{R}$, y su dotación de recursos $\omega_{i} \in \boldsymbol{R}_{+}^{\ell}$.

En un escenario simple, con dos consumidores $A$ y $B$, se parte del supuesto que estos dos clientes tienen dos enfermedades distintas, el $A$, padece de una enfermedad considerada como catastrófica como lo es la diabetes y $B$ acude al médico por enfermedades menores "gripa común"13". $A$ y $B$ pertenecen de igual manera, al mismo grupo etareo y están en el régimen de salud contributivo. En estos términos el conjunto $\ell$ mercancías del enfermo de diabetes es distinto del conjunto $\ell$ mercancías del consumidor de medicamentos de enfermedades menores. Para el consumidor $A$, la elasticidad precio de insulina es totalmente inelástica, es decir, que el consumidor estaría dispuesto a pagar a cualquier precio $p$ la misma cantidad dentro de su plan de consumo $x_{i}=\left(x_{i 1}, x_{i 2}, \cdots x_{i \ell}\right)$ correspondiente para aliviar el dolor causado por la esta enfermedad. Ahora el consumidor $B$, como existen un sinnúmero de medicamentos genéricos y comerciales para aliviar la gripa común, su elasticidad precio es más elástica.

13 Aunque estas generalmente se presentan con mayor frecuencia por fenómenos estacionales, lo que genera coaliciones en un periodo de tiempo.

\subsubsection{Coaliciones en el consumo de las demanda de salud}

Lo anterior deja ver que $A$ y $B$ tienen planes de consumo distintos, y por supuesto sus demandas dependen de su estado de salud. Ante esto, el SGSSS en su interior cuenta con lo que se ha denominado mecanismos de coaliciones, es decir, que las posibilidades de intercambio del un consumidor $A$, no se reducen a los acuerdos posibles con otro consumidor B. En estos términos, Villar (2006), señala que una coalición no es más que un subconjunto de consumidores.

Ahora, ¿qué pasaría si esta coalición fuera generalizada, o se ponderara al resto de los hogares que cuentan con enfermos de enfermedades catastróficas? Si fuera un sólo individuo, entonces $i \subset \mathbb{M}$, donde $\mathbb{M}$ corresponde a la gran coalición. Ante la gran coalición entonces $\mathbb{M}=1,2 ; \cdots, m$. Entonces, dada la coalición de enfermos $S$ de diabetes $S \subset \mathbb{M}$, sus planes de consumo estarían represetados por el conjunto de elección de $X_{i} \subset \boldsymbol{R}_{+}^{\ell}$ que los lleva al equilibrio de su estado de salud. En este caso $\left(x_{i}\right)_{i=1}^{m}$ en donde $x_{i}$ sería el medicamento, insulina, será la asignación factible para estos enfermos, si $\sum_{i=5} \omega_{i}$ Así pues, siguiendo a Villar, "una asignación es factible para una coalición $S$ si los intercambios que supone son posibles para los miembros de la coalición, sin tener que recurrir al resto de los consumidores"

Qué pasaría con $E=\left(\boldsymbol{R}_{+}^{\ell}, \geq_{i}, \omega_{i}\right)_{i=1}^{m}$, si se replica a los hogares que tienen pacientes con diabetes, entonces $E^{k}=\left(\boldsymbol{R}_{+}^{\ell}, z_{i}, \omega_{i}\right)_{i=1}^{m}$, y $E^{k}$ será una economía replicada de $E$, compuesta por $k$ consumidores idénticos con diabetes. Por idénticos se entiende que tienen el mismo plan de consumo $x_{i}=\left(x_{i 1}, x_{i 2}, \cdots x_{i \ell}\right)$ en donde $\left(x_{i}\right)_{i=1}^{m}$ les corresponderían las mismas funciones de utilidad y las mismas dotaciones $\omega_{i}$ dado su estado de gravedad. 
En estos términos, muchos de los problemas por los que atraviesa la demanda de mercancías que van a permitir la maximización del estado de salud, sujeto a una restricción presupuestaria dada, se necesitan, como lo orienta Escobar (2005), "que no es posible tener puntos de buen comportamiento sin exigirle a las funciones objeto de estudio propiedades que nos aseguren dicha globalidad”. En este sentido, se necesita que para el logro del máximo global único del estado de salud, que el conjunto de sus planes de consumo $X_{i} \subset \boldsymbol{R}_{+}^{\ell}$ sea convexo, cerrado y acotado. Si esto es así, debe cumplirse de acuerdo con la definición (Escobar, 2005, p. 1), si para cualquier $x_{1}, x_{2} \in X \mathrm{y}, \lambda \in[0,1], \lambda x_{1}+[1-\lambda] x_{2} \in X$.

\subsubsection{La demanda dinámica del estado de salud}

$\mathrm{Al}$ parecer, el estado de salud rompe con la ley de $\mathrm{Say}^{14}$, es decir, que en este mercado cada "demanda genera su propia oferta" ${ }^{15}$. De igual manera, las enfermedades son dinámicas y dependen en parte de la edad de las personas y del grupo al que pertenecen, por ejemplo de acuerdo con el Sistema General de Salud (Sinais) ${ }^{16}$, para el año 2005, la enfermedad que más defusiones causa en entre los 15 y 64 años es la "Diabetes mellitus" con 50,5\%, mientras que los menores de cinco años, son causadas por "Ciertas afecciones originadas en el periodo perinatal", las cuales causaron defunciones en el orden de 50,5 a nivel nacional. A continuación se presenta la siguiente figura que demuestra lo anterior.
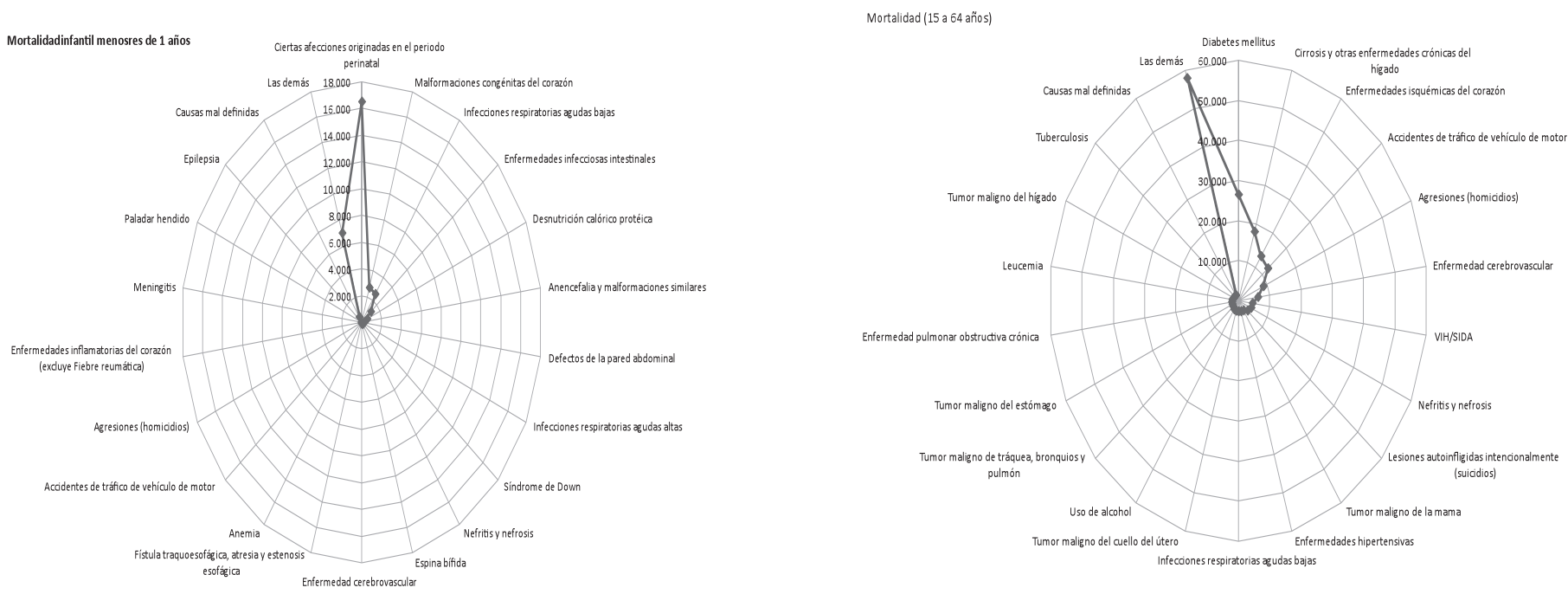

Figura 3. Tipos de enfermedad por edades.

Fuente: Anexo 1.

14 Say Jan Batista consideraba que cada oferta generaba su propia demanda.

15 Este es un planteamiento keynesiano.

16 [http://sinais.salud.gob.mx/acercade/index.html] 
Como puede observarse, las enfermedades muestran comportamientos dinámicos, es común que se diagnostiquen en los menores (niños) malformaciones genéticas, mientras que los mayores, en edad productiva, se diagnostique cirrosis (ver anexo 1).

De igual manera, la demanda en salud depende de las condiciones de las expectativas de vida. Esta dependerá de las condiciones de vida de "hoy y mañana", lo que obliga a que los planes de consumo y preferencias también cambien con el tiempo. En estos términos el plan de consumo estaría dado por un vector $x_{i}=[(x)]_{i}^{0}, x_{i}^{1}$, donde $x_{i}^{0} \in R_{+}^{\ell}$, situación que describe las mercancías disponibles para un subgrupo de la coalición hoy y $x_{i}^{1} \in R_{+}^{\ell}$, la demanda de mercancías disponibles para otro tipo de enfermedad, del mismo individuo pero en otro grupo distinto del primero. Esto quiere decir que su conjunto de mercancías convexas acotadas, estaría representada por: $x_{i}^{0}, x_{i}^{1} \in X$ y $\lambda \in[0,1], \lambda x_{i}^{0}+[1-\lambda] x_{i}^{1} \in X$. Al respecto Villar (2006) señala que:

En ocasiones se supone que la utilidad del consumidor es adictivamente separable en el tiempo, lo que significa que $\left.u_{i}[(x)]_{i}^{0}, x_{i}^{1}\right)=u_{i}^{0}\left(x_{i}^{0}\right)+\left[\left(u_{i}^{0}(x)\right]_{i}^{1}\right)$, donde $u_{i}^{j}: R_{+}^{k} \rightarrow R$ es una función que describe las preferencias del $i-$ ésimo consumidor en el periodo $t=0,1 \mathrm{La}$ función de utilidad puede variar de un período a otro, indicando los gustos o las necesidades que del individuo pueden cambiar con el tiempo (pueden verse afectadas por la edad, entre otras cosas).

Por tratarse de expectativas de vida saludables, se podría pensar en una aproximación de la realidad a través de un modelo de expectativas adaptables. Esto es: $Y_{t}=f\left(Z_{t}^{*}\right)$, donde $Y_{t}$ corresponde a la demanda de salud en el tiempo de un individuo, y $Z_{t}^{*}$ la expectativa de vida al nacer. Ahora, dado que la expectativa de vida no es observable, y si se parte del hecho que el individuo a lo largo de su vida no sufriera de ninguna enfermedad (catastrófica), se formula el siguiente modelo y su hipótesis de expectativas adaptativas:
$Y_{t}=\beta_{0}+\beta_{10} Z_{t}^{*}+\varepsilon_{t}$, su hipótesis está representada por $Z_{t}^{*}-Z_{t-1}^{*}=\lambda\left(Z_{t}-Z_{t-1}^{*}\right)$, donde está $Z_{t}^{*}, Z_{t-1}^{*} \in Z$ y $\lambda \in[0,1]$. $\mathrm{Al}$ solucionar la hipótesis se tiene que $0<\lambda<1$, es decir $\lambda \in[0,1],(1-\lambda)$ es la velocidad de ajuste, y la hipótesis será: $Z_{t}^{*}=\lambda Z_{t}+(1-\lambda) Z_{t-1}^{*}$. Ahora, $\lambda=0$, se esperaría que $Z_{t}^{*}=Z_{t-1}^{*}$. Asimismo, las expectativas serían estáticas, por lo que "las condiciones que prevalecen hoy se mantendrán en todos los períodos subsiguientes". (Gujarati, 2004), alcanzaría un máximo global único, toda vez que los valores futuros esperados se identifican con los valores actuales. Sí la vida es $\lambda=1$, entonces $Z_{t}^{*}=Z_{t}$, lo cual significa que las expectativas de vida se cumplen inmediatamente.

\subsubsection{La oferta}

En el SGSSS se observa en el esquema 2, la oferta de los bienes y/o servicios de salud, es realizado a través de entidades promotoras de salud (EPS) del régimen contributivo y las EPS del régimen subsidiado ${ }^{17}$. Estos agentes se encargan de la afiliación de los trabajadores formales, la cuenta propia y los pensionados, de igual manera, son las entidades administradoras de los recursos del régimen contributivo, mientras que las ARS afilian a la población considerada como pobre y vulnerable y los del régimen especial denominado vinculado. Estas últimas administran los recursos del régimen subsidiado. Los clientes del sistema reciben atención a través de las instituciones prestadoras de servicios (IPS), donde acuden los consumidores a recibir planes de consumos $x_{i}=\left(x_{i 1}, x_{i 2} \cdots x_{i \ell}\right)$ de acuerdo con el tipo de servicio y de enfermedad. Para suplir las dotaciones $\omega_{i}$, de cada consumidor, el SGSSS creó en un Plan Obligatorio de Salud (POS), de manera diferenciado para los dos regímenes contributivo y subsidiado. Dichos regímenes a través del tiempo deberían ser iguales, que deberían converger.

17 Antes de la Ley 1122 de 2007 se les denominaba las Administradoras del Régimen Subsidiado ARS. 
El teorema de la convergencia en un mercado competitivo sugiere de acuerdo con Villar (2006) que: "cuando una economía está compuesta por muchos agentes los equilibrios competitivos son una buena descripción de los resultados que cabe esperar para el funcionamiento de la economía" Ahora, de acuerdo con el ejemplo de los dos consumidores $A$ y $B$, en el mercado de salud $E^{k}=\left(R_{+}^{\ell}, z_{i k}, \omega_{i k}\right)_{i=1}^{m}$, desde la oferta se crearon monopolios en la provisión de medicamentos y oligopolios en la prestación de servicios de salud, por parte de las EPS.

En estos términos, si se parte de que la demanda genera sus propias ofertas, las funciones de producción y de costos que van a dar lugar a suplir las necesidades de la demanda del servicio, dependerán de las exigencias de las coaliciones de demandantes. Como toda empresa sus metas u objetivos son maximizar el beneficio, minimizando los costos de producción y de las acciones que se puedan tomar. En estos términos, el beneficio está dado por la resta entre los ingresos y los costos.

De acuerdo con la tecnología empleada, por ejemplo, con una función de producción tipo Cobb-Douglas dada por $f(K, L)=A K^{\alpha} L^{\beta}$, siendo el progreso tecnológico $A>0$. En teoría las empresas para encontrar su función de costo, considera Escobar (2005), tendrían que resolver el siguiente problema:

$\min w L+r K$ s.a $f(K, L)=A K^{\alpha} L^{\beta}=q$, donde $w$ y $r$ son los precios de los factores $K$ y $L$ son el trabajo y el capital. En otras ocasiones dependiendo de la elasticidad de sustitución, el productor asumirá funciones más especializadas y dependiendo de las necesidades del consumo (medicamentos de elasticidad precio totalmente inelásticos o elásticos), como funciones de elasticidad constantes CES, las cuales se formalizan como:
$Y=A\left[\delta K^{-\rho}+(1-\delta) L^{-\rho}\right]^{-\frac{v}{\rho}}$, , donde $Y$ representa la producción, $K$ es el insumo capital, $L=$ Insumo trabajo, $A=$ Tecnología. Por otra parte $\delta$ es el parámetro de distribución y es un valor $0<\delta<1$; $v$ corresponde al grado de homogeneidad y $\underline{v}$ explica la introducción explicita de factores que midan la escala.

Rosales et ál. (2004), señala que esta función presenta estricta concavidad cuando $A>0 ; 0<\delta<1 ; 0<v \leq 1$ y $\rho>-1$ y existirá estricta cuasiconcavidad. Si $A>0$ $0<\delta<1$ y $v>0$ y $\rho>-1$. Como la $\mathrm{CES}^{18}$ es una función no lineal en los parámetros, para su estimación, Greene (1998), señala que debe realizarse la aproximación de series de Taylor a la función transformada alrededor del punto $\rho=0$.

\subsubsection{El equilibrio: un asunto por resolver}

En este tipo de mercado, con toda esa serie de elementos señalados tanto en la oferta y la demanda, sus desarrollos son complejos. No obstante, el Estado se ha centrado en mantener el equilibrio financiero del SGSSS olvidándose de lo fundamental y el entronque entre los equilibrios macro y general. Este equilibrio financiero, se realiza a través del Fondo de Solidaridad y Garantías (FOSYGA), que es la entidad encargada de girar la UPC a las ARS y del proceso de compensación en las EPS, las cuales, a través del Sistema Financiero recaudan y giran al FOSYGA y éste envía o compensa con UPG, cuando la compensación es deficitaria. Se esperaría, según Chicaiza (2002), que existiera un equilibrio financiero en el SGSSS y que por ello, hay una exigencia que en el régimen contributivo, los beneficios sean iguales a los gastos $(I=G)$.

18 Meza (2009), "Econometría fundamental" realiza una estimación de esta función para la economía Española. En donde detalla todos estos procedimientos. 


\subsection{Equilibrio y núcleo de la economía: un asunto por resolver}

Dadas las interrelaciones microeconómicas de los agentes, el núcleo de la economía y su circuito económico (producción, consumo, acumulación, resto del mundo), se afectaría por los intercambios que se dan al interior SGSSS entre los planes de consumo $x_{i}=\left(x_{i 1}, x_{i 2} \cdots x_{i \ell}\right)$ de las coaliciones tanto oligopsonicas y monopsonicas, frente al conjunto de dotaciones $\sum_{i \in S} \omega_{i}$ que deben proveer los monopolios de medicamentos e instrumentales médicos y, los oligopolios en el aseguramiento por parte de las instituciones compuestas por las EPS y ARS. Uno de los equilibrios, se presentaría en el aspecto curativo de pacientes (clientes), el cual se representaría como coaliciones de enfermos, por ejemplo de diabetes, frente a la(s) compañía(s)

'09 87que ofrecen el medicamento insulina. De acuerdo con lo anterior la oferta total de estas mercancías estaría dada por $\sum_{i=1}^{m} \omega_{i k}$, las cuales deben ser iguales a las demandas agregadas de las coaliciones o subgrupos de agentes $S$ que requieran los medicamentos especializados, los exámenes de laboratorios y demás que den lugar al tipo de enfermedad. En estos términos se puede presumir que el plan de consumo de cada uno de los consumidores de la coalición estaría dado por $x_{i}=\omega_{i}$.

Ahora, si se considera que la función demanda de cada consumidor individual, en un mercado competitivo está dada por $d_{i}\left(p, M_{i}\right)$ en donde $p$ es el precio y $M_{i}$ corresponde a la riqueza ${ }^{19}$, entonces el plan de consumo agregado $d_{i k}\left(p, M_{i k}\right)$, corresponderá a los consumos de todos los consumidores, dada sus restricción presupuestarias y su capacidad de riqueza. En el caso del SGSSS, se asumie- ron el régimen contributivo y el subsidiado, que permiten diferenciar a los asegurados (consumidores) por nivel de ingreso. No obstante, lo que se pretende con estos regímenes que en el mediano plazo estos deberían converger, lo que concuerda con el teorema de la convergencia a partir del POS.

Este teorema depende, esencialmente, del hecho de que toda la asignación del núcleo de una economía replicada tiene la propiedad del tratamiento igualitario (todos los consumidores de un mismo tipo reciben los mismos bienes). Esta propiedad de tratamiento igualitario deja de cumplirse cuando aumentan los agentes de la economía con distinto número de consumidores de cada tipo (Villar, 2006).

La situación del precio de los medicamentos $p$, depende en parte de las negociaciones entre las multinacionales (resto del mundo), que en su gran mayoría son las proveedoras de medicamentos especializados, comerciales y genéricos y las EPS, que finalmente le trasladan a los planes de consumo de los consumidores y los hogares.

\section{Propuesta metodológica}

Esta reflexión busca encontrar elementos de la microfundamentación y la macrofundamentación de un sector que consideró la salud como una mercancía negociable en el mercado. De esta forma, la propuesta metodológica puede dar lugar a un encuentro entre el equilibrio micro con el macro. Esto es posible si se logra entroncar el SGSSS en el circuito económico, puesto que no se logra la armonía y no responden las relaciones funcionales que deben dar lugar a unos indicadores de calidad de vida satisfactorios, en lo relacionado con el estado de salud de los colombianos de manera plena, sin que tengan que recurrir a elementos judiciales para este fin.

19 La riqueza corresponde a todos los recursos que los individuos poseen como los derivados del trabajo, casa, libros, electrodomésticos entre otros. 


\subsection{Metodología}

Como se trata de una reflexión en un mercado en el que se rompen muchas reglas de la competencia perfecta y la ley de Say, encontrar el equilibrio que conduzca a determinar los impactos micro y macro en la institución hogares, causada por la ineficiencia del mercado y del Estado; para solucionar un problema de política pública en salud, uno de los modelos que podría dar respuesta es el de Equilibrio General Computable, en un ambiente walrasiano pero de competencia imperfecta.

En primera instancia, se propone la construcción de una Matriz de Contabilidad Social SAM. Esta matriz se construye de la siguiente manera:

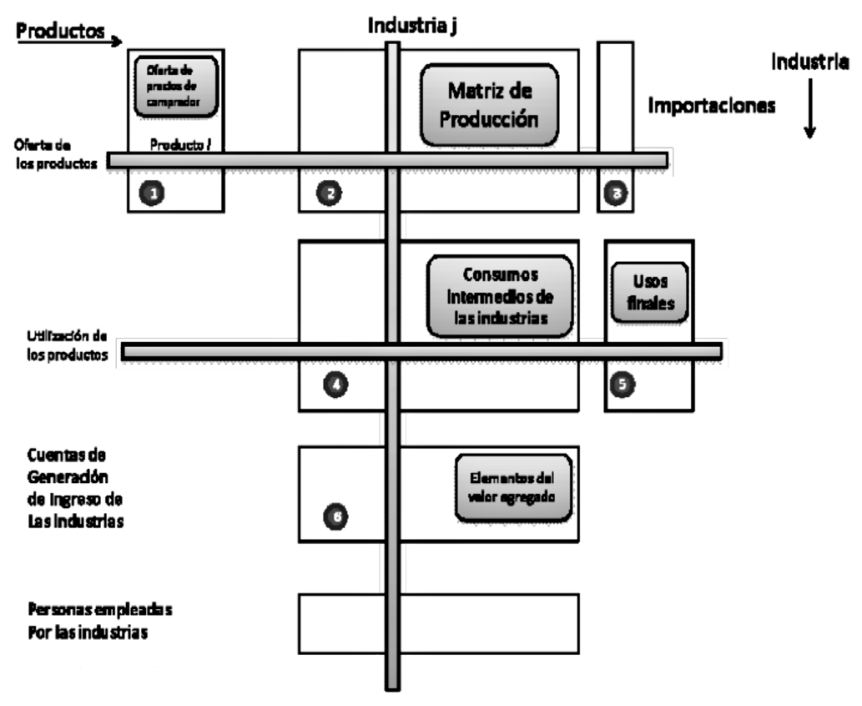

Figura 4. Cruces de matrices para construir la SAM macro.

Diseño Autor: Fuente Seruzier: 2003.

Asimismo, habría que construir en primera instancia una Matriz de Contabilidad Social (SAM) (Meza C, León, N
\& Acosta, C., 2009) ${ }^{20}$, a partir de las matrices de utilización, oferta y de equilibrio en un año base de análisis que se encuentran en el DANE. Su construcción tendría en cuenta, las metodologías ofrecidas por Seruzier (2003) quien orienta su uso a través de la figura 4 y, podría servir de base para su construcción.

La construcción de esta herramienta exige de una matriz cuadrada cuya información proviene del cruce de diferentes matrices (equilibrio general, oferta y utilización). El principio contable de la doble entrada que subyace a la estructura de la SAM, exige que para cada cuenta, los ingresos totales de cada fila sean iguales a los gastos totales de su columna. En otras palabras, la SAM sería la herramienta de contabilidad socioeconómica que serviría de base correlacionar en la estructura productiva de la economía, la distribución de ingresos y los gastos de las instituciones en un tiempo determinado. Con la construcción de la SAM, permite a través de una matriz técnica de Leontief, encontrar los multiplicadores técnicos de contabilidad social.

La construcción del MEGC recogerá todas las transacciones de los agentes económicos que se comportan de acuerdo con los principios de optimización microeconómica (Cicowiez et ál, 2004). La situación del SGSSS no se presenta bajo la estructura de competencia perfecta, una aproximación teórica-empírica, con los elementos del equilibrio general, son los Modelos de Equilibrios General Computable MEGG, cuya idea se centra a partir de la micro y macrofundamentación, encontrar interrelaciones entre los agentes.

En el MEGC se modelan, a partir de un escenario (software especializado), las características básicas y esenciales, bien sea, de un orden institucional o legal, del

20 La elaboración de una SAM, donde se presentan los fundamentos y elementos en la construcción de la misma. En esta oportunidad, para involucrar el género. 
comportamiento de los agentes económicos (familias, empresas, gobiernos), de tecnologías incorporadas en los procesos productivos (generalmente, estas ecuaciones se estiman a partir de funciones CES, Coob-Douglas, Leontieff o a las que haya lugar).

\subsection{Algunas aproximaciones}

El estudio que más se acerca a lo que se pretende realizar, es el de Ramírez et ál. ${ }^{21}$, quienes realizan una SAM para el análisis de políticas de seguridad social en salud, y cuyo propósito fue el de establecer las interrelaciones y transacciones de los agentes que componen el SGSSS, y en especial las que afectan la estructura financiera del Sistema. Por su parte Segura (2010), en un estudio realizado a la Secretaria de Desarrollo Económico, presenta algunos elementos de modelos dinámicos VAR, que pueden dar importancia al avance de este tipo de estimaciones.

Se encuentra que en Colombia no hay estudios que apunten a determinar los impactos micro y macroeconómicos del SGSSS, que por su ineficiencia, se manifestada en fallas de mercado que recaen sobre la institución hogares. Por esta razón, la reflexión pone de manifiesto que las ecuaciones de identidad macroeconómicas sean las que permiten encontrar a partir de la estructura económica, las ecuaciones que van a dar lugar al circuito económico y encontrar las relaciones de dependencia lineal o no lineal en la armazón estructural del modelo que entronque los equilibrios macro y general en un ambiente walrasiano, en una estructura tan compleja como es el SGSSS, y sus relaciones con el equilibrio micro y macroeconómico.

21 [http://www.urosario.edu.co/economia/documentos/pdf/bi89.pdf]

\section{Conclusiones}

La salud desde el enfoque teórico neoclásico, en el cual se considera como una mercancía negociada en el mercado y regulada por el Estado, es un Sistema que se preocupó por el equilibrio financiero del SGSSS, y por mostrar una mayor cobertura del mismo y, descuidó el equilibrio fundamental de un servicio público, cuyo objetivo es lograr el estado de salud y la calidad de vida de la población. De igual manera, el equilibrio del circuito macroeconómico dado por las relaciones funcionales que existenten entre las instituciones, hogar, empresa, Gobierno y resto del mundo, por la poca eficiencia del Estado en su papel de vigilancia y control, terminaron recargándose sobre la institución hogar.

Lo anterior no ha permitido la armonía o el equilibrio entre los elementos constitutivos del modelo implementado en el SGSSS, por el contrario, se quebrantan las relaciones matemáticas existentes que son las que dan lugar a las transacciones de bienes y servicios de salud. Al parecer, el problema funcional, puede ser la existencia de muchas ecuaciones, pero pocas variables por parte del Estado y el mercado para solucionar el problema del sector salud, lo que da lugar a que sea la ecuación de consumo de hogares de bienes y servicios de salud, la que tiene que responder por el resto de ecuaciones del sistema de ecuaciones simultaneas componentes del modelo del SGSSS.

No existen, o por lo menos no se ha encontrado el equilibrio que debe existir entre estado de salud y las funciones técnicas de producción por parte de las aseguradoras. Este tipo de mercado se soporta sobre de las enfermedades (males) que se presentan al interior de los hogares y de la sociedad; en estos casos se esperaría por parte de las EPS una respuesta oportuna y pertinente, sin que tenga que acudirse a medios legales como por ejemplo a la tutela. 
El modelo de salud, aunque caracteriza a la población por grupos etéreos, se podría tener un control sobre el tipo de enfermedad por grupo, no obstante, por ser un modelo que en el fondo se soporta sobre la oferta de males, enfermedades que aparecen de manera silenciosa, que les genera a las aseguradoras EPS, situaciones de incertidumbre que dan lugar a la afectación de sus funciones de producción. De igual manera, la presión que ejercen las coaliciones, por ejemplo de enfermos de enfermedades catastróficas, dan lugar que el equilibrio del SGSSS se vea afectado.

El modelo planteó como fin que las comunidades y las personas pudieran gozar de una calidad de vida, pese a que esa calidad de vida se ve afectada tanto por fallos de mercado como fallos del Gobierno, y no se garantice. Entre los fallos institucionales se encuentran la creación de leyes soportadas sobre mercados de derecho, "el que contamina paga", y entre los de mercado los bienes públicos preferentes y las externalidades, entre otros.

Todos los elementos tenidos en cuenta para la reflexión son el fruto a someter, el estado de salud de la población a las leyes del mercado y condicionar la calidad de vida a un mercado de derechos. Encontrar un equilibrio exige en primera instancia, la garantía de un ambiente sano, de la prevención antes que la curación y buscar otras alternativas de solución distintas a la garantía del equilibrio financiero del SGSSS. El verdadero equilibrio y el fin de cualquier sistema de salud se logra cuando las instituciones hogar, empresa, Gobierno y resto del mundo, en conjunto asuman riesgo para el logro del estado equilibrado de la salud y no como se pretende en Colombia, que sea la institución hogares la que cargue con el peso de la ineficiencia de las demás instituciones componentes del circuito económico.

\section{Referencias}

Escobar, A. (2005). Economía matemática. 2a ed. Bogotá: Ediciones Uniandes, Alfa Omega.

Castaño A. (2000). Medicina, ética y reforma a la salud: hacia un nuevo contrato social con la profesión médica. Bogotá: Ecoe Editores.

Dagum, G. \& Bee, E. (1983). Introducción a la econometría. México: Editorial Siglo XXI.

Dornbusch, R., Stanley, F. \& Startz R. (2004). Macroeconomía. $9^{a}$ ed., Bogotá: McGraw-Hill.

Cicowiez, M. \& Di, L. (2004). Economía computacional: equilibrio general computado. La Plata: Departamento de Economía. Facultad de Ciencias Económicas, Universidad de la Plata. Trabajo docente, No 7.

Chicaisa, L. (2002). El mercado de la salud en Colombia y la problemática de alto costo. En Problemas del desarrollo, Vol 33, No. 131 X -XII.

Gujarati, D. (2004). Econometría. $4^{a}$ ed. México: McGraw Hill.

Greene, W. (1998). Análisis econométrico. $3^{\mathrm{a}}$ ed. Madrid: Editoral Prentice.

Keynes, J. (2000). Teoría general de la ocupación el interés y el dinero. $5^{\mathrm{a}}$ ed. Bogotá: Fondo de Cultura Económica.

Lora, E. (2005). Técnicas de medición económica. Bogotá: Tercer Mundo Editores.

Mas-Collel, W. \& Green, J. (1995). Microeconomic theory Oxford: Oxford University. 
Monsalve, S. (1999). Introducción al equilibrio en economía. $1^{\text {a }}$ ed. Bogotá: Facultad de Ciencias Económicas, Universidad Nacional de Colombia.

Meza, C., León, N. \& Acosta, C. (2009). Elementos y fundamentos para la construcción de una SAM con enfoque de género. En Equidady desarrollo, (comp.) López, A., Meza, C. \& Sanabria, N. Bogotá: Ediciones Unisalle.

Meza, C. (2009). Econometría fundamental. $1^{\text {a }}$ ed. Bogotá: Ediciones Unisalle.

Meza, C. (2010). Econometría de series de tiempo. Bogotá: Elementos y Fundamentos. Mimeo, Unisalle.

Nicholson, W. (2007). Teoría microeconómica: principios básicos y ampliaciones. $9^{\mathrm{a}}$ ed. Mexico: Cengage Learning.

Ramírez, M., Yepez, F. \& Karl, C. Construcción de una matriz de contabilidad social para el análisis de políticas de seguridad social en salud. Documento en http://www.urosario.edu.co/economia/documentos/pdf/bi89.pdf

Rosales, R., Apaza, M. \& Bonilla, J. (2004). Economía de la producción de bienes agrícolas. Teoría y aplicaciones. Bogotá: Universidad de los Andes.

Segura, J. (2010). Modelo de equilibrio general dinámico de Bogotá. Bogotá.
Séruzier, M. (2003). Medir la economía de los países, según el sistema de cuentas nacionales. CEPAL, Editorial Alfaomega.

Stiglitz, J. (2002). La economía del sector público. $3^{\text {a }}$ ed. España: Antoni Bosch Editores.

Valenzuela, R. (2006). El que contamina paga. Red Verde Cajamarca. En http://caballeroredverde.blogspot. com/2006/09/el-que-contamina-paga.html

Varian, H. (s.f.). Microeconomía intermedia: un enfoque intermedio. $4^{\mathrm{a}}$ ed. Antoni Bosch Editores.

Varian, H. (1992). Análisis microeconómico. $3^{\mathrm{a}}$ ed. Barcelona: Antoni Bosch Editores.

Villar, A. (2006). Microeconomía. $1^{\text {a }}$ ed. España: McGrawHill.

\section{Leyes consultadas}

Constitución Política de Colombia (1991). Ley 100 de 1993. Sistema de Seguridad Social Integral.

Constitución Política de Colombia (1991). Ley 99 de 1993 SINA.

Constitución Política de Colombia (1991). Ley 643 de 2001.

Constitución Política de Colombia (1991). Ley 715 de 2001. 


\section{Anexo 1}

\section{Principales causas de mortalidad en edad productiva de 15 a 64 años, 2005.}

\begin{tabular}{|c|c|c|c|c|c|c|}
\hline \multicolumn{7}{|c|}{ Nacional } \\
\hline Orden & $\begin{array}{c}\text { Clave CIE 10a. } \\
\text { Rev. }\end{array}$ & Descripción & Defunciones & $\operatorname{Tasa}^{1 /}$ & & $\%$ \\
\hline & A00-Y98 & Total & 181.719 & 264,0 & 100,0 & \\
\hline 1 & E10-E14 & Diabetes mellitus & 26.385 & 38,3 & 14,5 & \\
\hline 2 & $\begin{array}{l}\mathrm{K} 70, \mathrm{~K} 72.1, \mathrm{~K} 73 \\
\mathrm{~K} 74, \mathrm{~K} 76\end{array}$ & $\begin{array}{l}\text { Cirrosis y otras enfermedades crónicas del } \\
\text { hígado }\end{array}$ & 17.872 & 26,0 & 9,8 & \\
\hline 3 & I20-I25 & Enfermedades isquémicas del corazón & 13.123 & 19,1 & 7,2 & \\
\hline 4 & 21 & $\begin{array}{l}\text { Accidentes de tráfico de vehículo de } \\
\text { motor }\end{array}$ & 12.245 & 17,8 & 6,7 & \\
\hline 5 & X85-Y09, Y87.1 & Agresiones (homicidios) & 8.604 & 12,5 & 4,7 & \\
\hline 6 & I60-I69 & Enfermedad cerebro vascular & 6.178 & 9,0 & 3,4 & \\
\hline 7 & B20-B24 & VIH/SIDA & 4.445 & 6,5 & 2,4 & \\
\hline 8 & N00-N19 & Nefritis y nefrosis & 4.331 & 6,3 & 2,4 & \\
\hline 9 & X60-X84, Y87.0 & $\begin{array}{l}\text { Lesiones autoinfligidas intencionalmente } \\
\text { (suicidios) }\end{array}$ & 3.781 & 5,5 & 2,1 & \\
\hline 10 & C50 & Tumor maligno de la mama & 2.906 & 4,2 & 1,6 & \\
\hline 11 & $\mathrm{I} 10-\mathrm{I} 15$ & Enfermedades hipertensivas & 2.788 & 4,0 & 1,5 & \\
\hline 12 & J10-J18, J20-J22 & Infecciones respiratorias agudas bajas & 2.665 & 3,9 & 1,5 & \\
\hline 13 & C53 & Tumor maligno del cuello del útero & 2.641 & 3,8 & 1,5 & \\
\hline 14 & F10, G31.2 & Uso de alcohol & 2.614 & 3,8 & 1,4 & \\
\hline 15 & C33-C34 & $\begin{array}{l}\text { Tumor maligno de tráquea, bronquios y } \\
\text { pulmón }\end{array}$ & 2.251 & 3,3 & 1,2 & \\
\hline 16 & C16 & Tumor maligno del estómago & 2.183 & 3,2 & 1,2 & \\
\hline 17 & J40-J44, J67 & $\begin{array}{l}\text { Enfermedad pulmonar obstructiva } \\
\text { crónica }\end{array}$ & 2.115 & 3,1 & 1,2 & \\
\hline 18 & C91-C95 & Leucemia & 1.993 & 2,9 & 1,1 & \\
\hline 19 & $\mathrm{C} 22$ & Tumor maligno del hígado & 1.647 & 2,4 & 0,9 & \\
\hline 20 & A15-A19, B90 & Tuberculosis & 1.639 & 2,4 & 0,9 & \\
\hline & R00-R99 & Causas mal definidas & 1.583 & 2,3 & 0,9 & \\
\hline & & Las demás & 57.730 & 83,9 & 31,8 & \\
\hline
\end{tabular}




\section{Č́lírít 16}

1/ Tasa por 100,000 habitantes

No se incluyen defunciones de residentes en el extranjero

2/ V02-V04 (.1, .9), V09.2-V09.3, V09.9, V12-V14 (.3-.9), V19.4-V19.6, V20-V28 (.3-.9), V29-V79 (.4-.9), V80.3-V80.5, V81.1, V82.1, V83V86(.0-.3), V87.0-V87.8, V89.2, V89.9, Y85.0

Fuente: Elaborado a partir de la base de datos de defunciones INEGI/Secretaría de Salud. Dirección General de Información en Salud. CONAPO, 2002. Proyecciones de la Población de México, 2000 - 2050. 


\section{Principales causas de mortalidad en edad productiva (menores de un año), 2005}

\begin{tabular}{|c|c|c|c|c|c|}
\hline \multicolumn{6}{|c|}{$\begin{array}{ll} & \\
\text { NACIONAL }\end{array}$} \\
\hline \multirow[t]{2}{*}{ Orden } & \multirow{2}{*}{$\begin{array}{l}\text { Glave CIE 10a. Rev. } \\
\text { A00-Y98 }\end{array}$} & \multirow[t]{2}{*}{ Descripción } & \multirow{2}{*}{$\begin{array}{l}\text { Defunciones } \\
32.590\end{array}$} & \multirow{2}{*}{$\begin{array}{l}\text { Tasa }{ }^{1 /} \\
1663,6\end{array}$} & \multirow{2}{*}{$\begin{array}{c}\% \\
100,0\end{array}$} \\
\hline & & & & & \\
\hline 1 & P00-P96 & Ciertas afecciones originadas en el periodo prenatal & 16.448 & 839,6 & 50,5 \\
\hline 2 & Q20-Q24 & Malformaciones congénitas del corazón & 2.688 & 137,2 & 8,2 \\
\hline 3 & J10-J18, J20-J22 & Infecciones respiratorias agudas bajas & 2.490 & 127,1 & 7,6 \\
\hline 4 & A00-A09 & Enfermedades infecciosas intestinales & 1.200 & 61,3 & 3,7 \\
\hline 5 & E40-E46 & Desnutrición calórica proteica & 590 & 30,1 & 1,8 \\
\hline 6 & Q00 & Anencefalia y malformaciones similares & 307 & 15,7 & 0,9 \\
\hline 7 & Q79.2-Q79.5 & Defectos de la pared abdominal & 206 & 10,5 & 0,6 \\
\hline 8 & J00-J06 & Infecciones respiratorias agudas altas & 179 & 9,1 & 0,5 \\
\hline 9 & Q90 & Síndrome de Down & 168 & 8,6 & 0,5 \\
\hline 10 & N00-N19 & Nefritis y nefrosis & 120 & 6,1 & 0,4 \\
\hline 11 & Q05,Q07,0 & Espina bífida & 110 & 5,6 & 0,3 \\
\hline 12 & I60-I169 & Enfermedad cerebro vascular & 108 & 5,5 & 0,3 \\
\hline 13 & Q39.0Q39.3 & Fístula traquosofagica, atresia y estenosis esofágica & 106 & 5,4 & 0,3 \\
\hline 14 & d50-d64 & Anemia & 96 & 4,9 & 0,3 \\
\hline 15 & 2/ & Accidentes de tráfico de vehiculo automotor & 75 & 3,8 & 0,2 \\
\hline 16 & X85-Y09,Y87,1 & Agresiones (homicidios) & 69 & 3,5 & 0,2 \\
\hline 17 & I30-I33,I38,I40,142 & $\begin{array}{l}\text { Enfermedades inflamatorias del corazón (excluye fiebre reumá- } \\
\text { tica) }\end{array}$ & 66 & 3,4 & 0,2 \\
\hline 18 & A39,G00,G03 & Meningitis & 65 & 3,3 & 0,2 \\
\hline 19 & Q35-Q37 & Paladar hendido & 53 & 2,7 & 0,2 \\
\hline \multirow[t]{3}{*}{20} & G40-G41 & Epilepsia & 52 & 2,7 & 0,2 \\
\hline & R00-R99 & Causas mal definidas & 461 & 23,5 & 1,4 \\
\hline & & Las demás & 6.933 & 353,9 & 21,3 \\
\hline \multicolumn{6}{|c|}{ 1/ Tasa por 100,000 habitantes } \\
\hline \multicolumn{6}{|c|}{ No se incluyen defunciones de residentes en el extranjero } \\
\hline \multicolumn{6}{|c|}{$\begin{array}{l}\text { 2/ V02-V04 (.1, .9), V09.2-V09.3, V09.9, V12-V14 (.3-.9), V19.4-V19.6, V20-V28 (.3-.9), V29-V79 (.4-.9), V80.3-V80.5, V81.1, V82.1, } \\
\text { V83-V86(.0-.3), V87.0-V87.8, V89.2, V89.9 , Y85.0 }\end{array}$} \\
\hline \multicolumn{6}{|c|}{$\begin{array}{l}\text { Fuente: Elaborado a partir de la base de datos de defunciones INEGI/Secretaría de Salud. Dirección General de Información en } \\
\text { Salud. }\end{array}$} \\
\hline
\end{tabular}

CONAPO, 2002. Proyecciones de la Población de México, 2000 - 2050. 